\title{
Direct Imaging of Point Defects in a Quasicrystal by Cs-Corrected Ultrahigh-Resolution $300 \mathrm{kV}-\mathrm{STEM}$
}

Eiji Abe* and Stephen J. Pennycook**

*Materials Engineering Laboratory, National Institute for Materials Science, 1-2-1 Sengen, Tsukuba 305-0047, Japan

**Condensed Matter Sciences Division, Oak Ridge National Laboratory, Oak Ridge, TN 37831, USA

Z-contrast scanning transmission electron microscope (STEM) observations of a quasicrystal have provided significant insights into the veiled structural details [1]; however, the resolution is still not sufficient to reveal a total structure including relatively low-Z atoms. To investigate further details in particular on local disorders, we here use a spherical aberration (Cs) corrected 300kV-STEM (VG-HB603U with Nion corrector, at ORNL), which has in fact achieved a sub-Å resolution [2].

It is obvious that the image becomes much clearer after Cs correction; see the image of a decagonal $\mathrm{Al}_{72} \mathrm{Ni}_{20} \mathrm{Co}_{8}$ shown in Fig. 1. Significantly, even the Al atomic columns, not only the heavier Ni or Co columns, are now seen clearly as weak bright spots even under the scattering amplitude sensitive Z-contrast mode. After a careful analysis with the aid of maximum-entropy deconvolution processing and image simulations, we find that chemical and occupational disorders are significant around the center of the 2nm-scale cluster (Fig. 2), a building unit of the $\mathrm{Al}_{72} \mathrm{Ni}_{20} \mathrm{Co}_{8}$ structure [1]. Occurrence of such localized disorder can be reasonably interpreted according to phason-related atomic behaviors [3]. With respect to achievable performance of the present STEM, it is remarkable that even the Al atomic sites, which are not fully-occupied and separated less than $1 \AA$ distance, have now become detectable. Change of local electronic structures will also be discussed through atomic column-by-column electron energy loss spectroscopy (EELS).

[1] E. Abe, Y. Yan and S. J. Pennycook, Nature Materials, 3 (2004) 759.

[2] P.D. Nellist et al.: Science, 305 (2004) 1741.

[3] E. Abe, S.J. Pennycook and A.P. Tsai, Nature 421 (2003) 347.

[4] We acknowledge K. Ishizuka for helpful discussions on deconvolution processing. This work was supported by USDOE under contract DE-AC05-00OR22725 managed by UT-Battelle, LLC. 


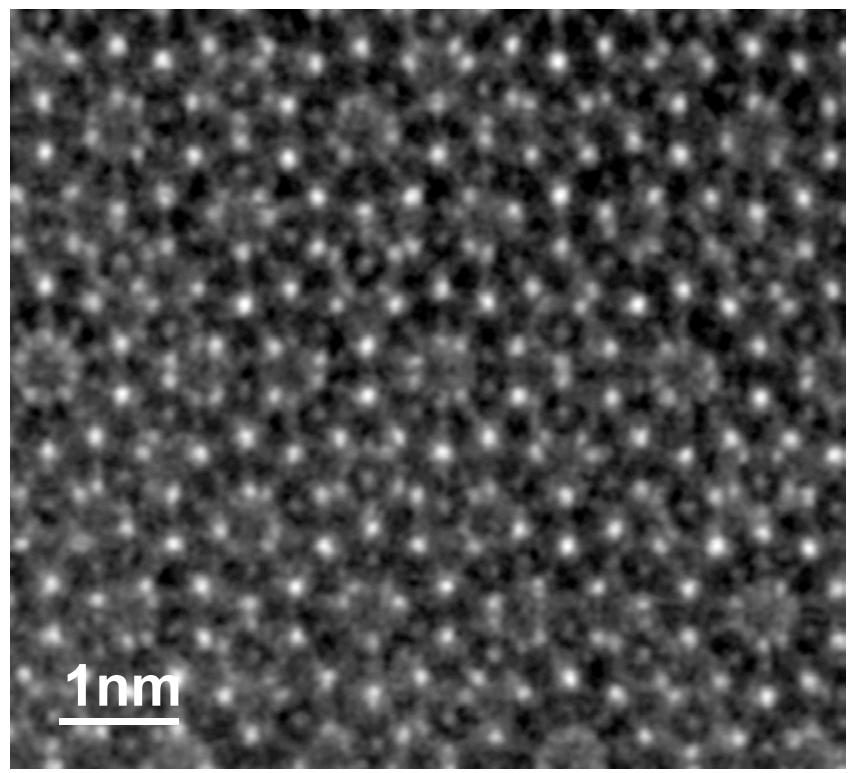

Fig. 1 Z-contrast image of the $\mathrm{Al}_{72} \mathrm{Ni}_{20} \mathrm{Co}_{8}$ decagonal quasicrystal, taken by the Cs-corrected 300kV-STEM (VG-HB603U with Nion corrector, at ORNL).
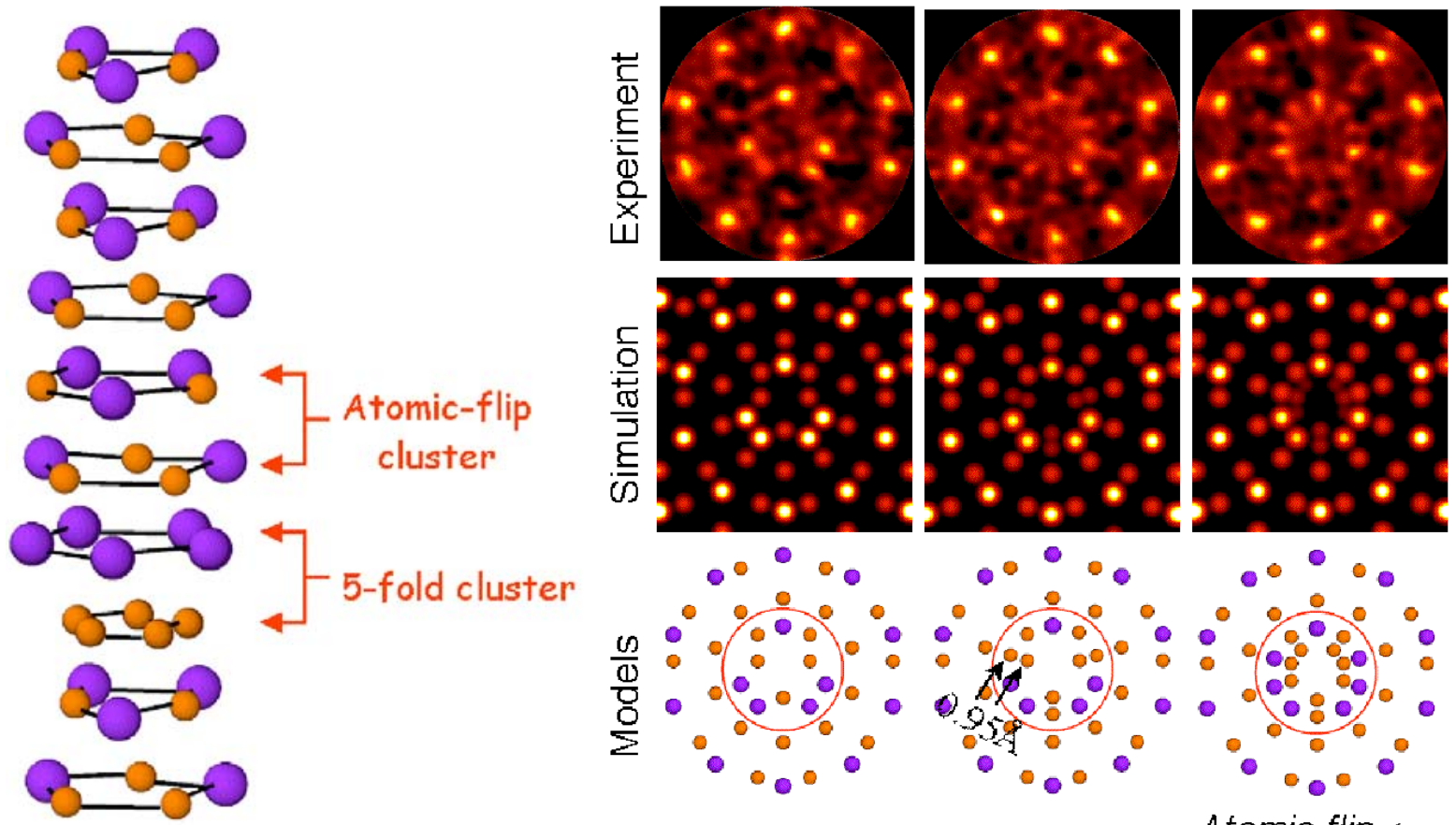

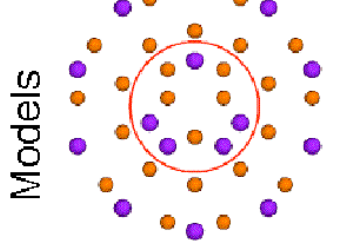

Order

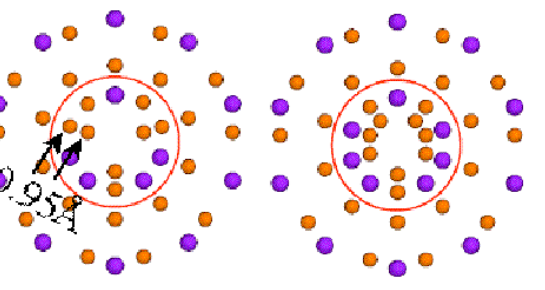

Atomic flip
Atomic flip + 5 -fold cluster

Fig. 1 Phason-related point defects (displacive, occupational and chemical disorders) observed around a center of the decagonal clusters in $\mathrm{Al}_{72} \mathrm{Ni}_{20} \mathrm{Co}_{8}$ quasicrystal. 